\title{
Human-Robot Collaboration Acceptance Model: Development and Comparison for Germany, Japan, China and the USA
}

\author{
Christina Bröhl $^{1}$ (D) Jochen Nelles ${ }^{1}$ (D) Christopher Brandl $^{1}$ (D) Alexander Mertens ${ }^{1}$ (D) Verena Nitsch $^{1}$ (D)
}

Accepted: 12 September 2019 / Published online: 5 November 2019

(c) The Author(s) 2019

\begin{abstract}
The use of robots in the national economy - especially in industrialized countries—is growing. At the same time, the interdependency between humans and robots is getting increasingly closer: they are engaging in direct contact with each other as more and more organizations let robots and humans work hand-in-hand. One factor that predicts successful human-robot interdependency is the acceptance of the robot by the human. Generally, only when an innovative assistive working system covers human needs and expectations, it is perceived to be useful and hence accepted. Furthermore, it has been found that cultural context has an impact on human-robot interaction, as people feel more comfortable interacting with a robot in a culturally normative way. Therefore this paper aims at presenting a human-robot collaboration acceptance model (HRCAM) with regard to the collaboration between humans and robots that is based on prior acceptance models, while also considering technology affinity and ethical, legal and social implications. Additionally, similarities and differences in robot acceptance are shown for four selected countries_-both in comparison to the overall human-robot collaboration acceptance model and between the countries. The HRCAM additionally shows which variables influence perceived usefulness and perceived ease of use, and thus behavioral intention to use and use behavior. A further distinction is made between anchor variables, which can be influenced in the long term, and adjustment variables, which can be influenced in the short to medium term. The model therefore offers practitioners in the field of human-robot collaboration recommendations to increase the acceptance of robots.
\end{abstract}

Keywords Technology acceptance $\cdot$ Human-robot interaction $\cdot$ Human-robot cooperation · Human-machine interaction · Cross-cultural differences · TAM

\section{Introduction}

Since the middle of the 20th century, robots have become a vital part of today's production industry [1, 2]. The latest development of robotics leads away from robots as a component of fully automated manufacturing processes towards

\section{Christina Bröhl \\ c.broehl@iaw.rwth-aachen.de \\ Jochen Nelles \\ j.nelles@iaw.rwth-aachen.de \\ Christopher Brandl \\ c.brandl@iaw.rwth-aachen.de \\ Alexander Mertens \\ a.mertens@iaw.rwth-aachen.de \\ Verena Nitsch \\ v.nitsch@iaw.rwth-aachen.de}

1 Institute of Industrial Engineering and Ergonomics, RWTH Aachen University, Bergdriesch 27, 52062 Aachen, Germany processes in which human and robot work closely together at the same place and time. The latest generation of robots, for example, include the ability to recognize the proximity of a human as well as contacts and their intensity and to adapt flexibly to them [3]. Although industrial robots are first and foremost "working machines", they include social aspects (e.g. the appearance of being a co-worker) which have to be considered and taken into account in an implementation. Thus, the question of designing ergonomic workplaces where the human and robot can accomplish a task together in a collaborative operation is becoming increasingly important. In accordance with the definition in ISO 10218-1 "Robots and robotic devices-Safety requirements for industrial robots-Part 1: Robots" from the International Standardization Organization [4] and ISO/TS 15066 [5], the term collaboration is used in this paper as a "state in which purposely designed robots work in direct cooperation with a human within a defined workspace". However, any erroneous behavior of the robot could result in serious injury to the human 
so the collaboration between human and robot has to be designed to be reliable and secure with regard to both hardware components as well as human cognition. In contrast, the term human-robot interaction is a general description of the interdependency between humans and robots.

It is predicted that by 2019 industrial robots worldwide will achieve a level of growth more than twice as high as was the case in 2010. Especially in industrialized countries, robots are used extensively in the national economy [6-8]. For instance, according to IFR estimates, there are 314 robots per 10,000 working persons in the national economy of Japan, followed by the Federal Republic of Germany (hereinafter abbreviated as Germany) (292 robots per 10,000 working persons), the United States of America (hereinafter abbreviated as USA) (164 robots per 10,000 working persons) and the People's Republic of China (hereafter abbreviated as China) (36 robots per 10,000 working persons). Furthermore, these countries have the biggest industrial robot sales. With 67,000 robot sales in 2015, China sold the most industrial robots worldwide, followed by Japan with 35,000 industrial robot sales, the USA with 27,000 industrial robot sales, and Germany with 20,000 robot sales [8]. Robots are no longer only used in large corporations, but due to simpler programming and lower costs more and more SMEs will introduce robots in the next few years, which will further increase sales. While most of these robots do not share a workspace with human workers, it stands to reason that a similar trend of propagation will likely be observed for collaborative robots, as the required technologies become increasingly cheaper and more robust.

\subsection{History in Technology Acceptance Research}

There are a number of different models for measuring technology acceptance. Rogers' diffusion theory from 1962 is fundamentally regarded as the starting point of this field of research. This theory proposes a five-step model, beginning with an awareness of a new technology and leading to its confirmation [9]. The next essential step regarding technology acceptance is Davis' Technology Acceptance Model (TAM) from 1989 [10, 11]. It forecasts the acceptance and corresponding use of information technologies. More precisely, this model is based on the fundamental assumption that the behavioral intention leads to actual behavior. The behavioral intention, in turn, depends on two variables - the perceived ease of use and the perceived usefulness. The perceived usefulness is defined as "the degree to which a person believes that using a particular system would enhance his or her job performance", whereas the perceived ease of use is defined as "the degree to which a person believes that using a particular system would be free from effort" [10]. Some studies measuring the acceptance of robots refer to the work of Davis. These studies add other variables to the original model, such as personal implications (social norm, voluntariness of usage and image) and job-related variables (job relevance, output quality, result demonstrability and experience) (TAM 2 by Venkatesh and Davis [12]). Another noteworthy approach is the Unified Theory of Acceptance and Use of Technology (TAM 3 by Venkatesh and Bala [13]), which aims at combining existing models of user acceptance of information technologies. In addition, Venkatesh and Bala [13] added variables to the TAM 3 model that relate to the anchoring and adjustment of human decision-making processes (computer self-efficacy, perception of external control, computer anxiety, computer playfulness, perceived enjoyment and objective usability). So far, however, acceptance models have not been adapted to the context of human-robot cooperation in an industrial setting and therefore neglect aspects to be considered important for this context.

The research that is presented in this paper therefore aimed at developing a human-robot collaboration acceptance model to investigate the acceptance of human-robot collaboration in an industrial work setting. To the knowledge of the authors, cross-cultural technology acceptance in the context of industrial robotics has not been explored so far with regard to collaboration but only for interaction variables. These results are summarized in the following paragraph.

\subsection{Cross-Cultural Differences in Human-Robot Interaction}

In the past few years, the interest of researchers to explore intercultural or cross-cultural acceptance of robots has grown. Transnational studies in this field were conducted within a Western or Eastern/Eastern-Asian cultural area, as well as cross-cultural studies between Western and Eastern countries, which will be described in more detail in the following. In the studies presented below, aspects such as direct versus indirect communication style, attitudes towards robots, anthropometry, robot type, and trust are discussed.

In general, it has been found that cultural background has an impact on human-robot interaction. Therefore, people feel more comfortable when interacting with a robot that behaves in a culturally normative way [14-18]. Crosscultural communication suggests that people thinking in Western patterns favor more direct forms of communication, whereas Eastern-influenced people prefer an indirect style of communication. Hall [14] introduced the idea of low and high context cultures, whereby low context refers to cultures in which communications are explicit and require little interpretation to understand the content. High context cultures, on the other hand, are those in which content is less central and deciphering of contextual cues is required for accurate decoding. Although people tend to use both forms of communication, one generally dominates in a given culture [14]. 
Generally, the results show that participants are more likely to accept the recommendations of a robot that speaks in more culturally normative ways. This illustrates that small adoptions to cultural preferences already have a positive effect on the whole interaction with a robot $[18,19]$. Westerners (those who live in the USA and Europe are more likely to accept the robot's recommendation and evaluate it more positively when the robot uses an explicit communication style, whereas Easterners (specifically, the Chinese) are more likely to accept robots and evaluate them more positively when they use an implicit communication style [16, 19]. Wang also shows that when collaborating in a human-robot team, Chinese participants were more likely to change their decision based on the robot's advice when the robot communicated implicitly. American participants were more likely to do so when the robot communicated explicitly [18]. Consequently, the communication types that are used in a society and by a robot influence the acceptance of recommendations provided by a robot $[18,19]$.

Cultural differences can also have an impact on human-human interaction. Based on a Taiwanese-German management team, Mahadevan [20] showed that tacit cultural differences can directly affect the team. In this study, cultural patterns were more general rather than contextual. In terms of comfort, the results show that people feel more comfortable when interacting with a robot that behaves in a more culturally normative way. Evers et al. [15] found that when a robotic assistant was characterized as a group member, Chinese subjects reported feeling more comfortable compared with Americans.

Moreover, Bartneck et al. [21] studied cultural differences in attitudes towards robots and found different attitudes depending on the cultural background of the subjects. In this cross-cultural study, the attitude of Dutch, Chinese and Japanese subjects using the "Negative Attitude towards Robots Scale" (NARS) questionnaire was examined. In contradiction to the popular belief that Japanese love robots, the results show that the Japanese are significantly more concerned with the impact that robots might have on society. A possible explanation could be that through their high exposure to robots, the Japanese are more aware of the robots' abilities and also their lack thereof [21]. In a follow-up study conducted by Bartneck et al. [22], different appearances of robots were examined, which indicated that American subjects rated a robot as more favorable when it was more anthropomorphic, while Japanese subjects showed the opposite trend. In general, the Japanese do not seem to have significantly more positive attitudes and positive assumptions towards robots than Europeans [23]. By using the NARS questionnaire, Bartneck et al. [22] found that the Japanese are even significantly more concerned with the impact that robots might have on society than Westerners (e.g. Americans and the Dutch). A possible explanation could be related to their higher exposure to robots e.g. in real life, but predominantly through the Japanese media [21-23]. Therefore, it is assumed that the Japanese have more robot-related experiences than the Chinese, Germans or Americans. However, Nomura et al. [24] found out that UK citizens felt more negative toward humanoid robots than did the Japanese when using the "Frankenstein Syndrome Questionnaire" (FSQ).

In addition, several studies have been conducted on the subject of trust. After interacting with a robot in a judgement task, Chinese participants evaluated the robots as being more trustworthy than German participants did [19]. When collaborating in a preference decision-making task together with a robot, Chinese participants were more likely to report trusting the robot than Americans did [18]. After interacting with a robot in four different scenarios (teaching, guide, entertainment and security) Germans rated the robot less trustworthy than Chinese and Koreans [25]. A study conducted by Haring [26], showed that Australians rated the robot more trustworthy after an economic trust game than Japanese participants did.

Cultural differences were also found concerning the evaluation of the robot after interaction (e.g. [18, 19, 25-27]) or after watching human-robot interaction (e.g. videos) (e.g. [15]). For instance, people with an Eastern cultural background (e.g. Chinese, Koreans and Japanese) rated the robot higher in animacy and anthromorphism [15, 18, 27], likeability $[19,25]$, trust $[18,19]$, perceived intelligence [27] and perceived safety $[26,27]$ than people with a Western cultural background (e.g. Americans, Germans and Australians). Following these results, it is assumed that Chinese and Japanese subjects evaluate the robot more positively after becoming more familiarized with it. Moreover, Easterners (Chinese and Koreans) show more engagement while interacting with a service robot in collaborative tasks than Westerners (e.g. Germans). Compared with German subjects, Chinese and Korean subjects perceived the service robots used in the experiment to be more likeable, satisfactory and trustworthy, and they had higher dedication with the robot. This finding is consistent with a previous study by Bartneck et al. [21] showing that German subjects had more anxiety and more concern about the robot's negative influence than Chinese subjects did [25].

Cultural differences were also found regarding future intentions to use a robot in a collaborative situation after watching a video in which a human and robot perform a collaborative task together [28] or after direct human-robotinteraction [29]. Indeed, these differences were found within Europeans (British and Italians or Germans and Dutch).

However, the evaluation of the robot can also differ regarding the type of robot in terms of the autonomy level. For example, the Japanese assume autonomy of humanoid robots more strongly than Koreans or Americans [30, 31]. In contrast, Lee et al. found that American subjects expected 
domestic robots to have high levels of autonomy [32]. Thus, it is expected that there are cultural differences regarding the robot's type (active vs. passive robot).

Previous studies have been mostly limited to measuring single aspects of cultural differences regarding the acceptance of social robots in a few countries. So far, to the best knowledge of the authors, there are no comparable studies measuring cross-cultural differences in industrial contexts. Moreover, a significant body of work is conducted mainly with test subjects recruited from universities (e.g. [18, 21, 29-31]) or unspecified test subjects without a precise description (e.g. [16, 23, 24, 32]). As far as we know, there are no studies measuring the acceptance of industrial workers. Therefore, the transferability of the available study result$\mathrm{s}$-with findings on social robots and test subjects from the university or everyday life-to industrial robots as well as work systems and test subjects from the industrial work environment is limited.

In short, based on the differences found in the attitudes towards robots in earlier studies (which compared Easterners and Westerners), this study aims at studying human-robot collaboration using Germany, Japan, China, and the USA as an example. The specific investigation of these four countries offers the potential to investigate the previously assumed dichotomy between a western and eastern culture in a more selective way and to identify possible causes for different technology acceptance more precisely. One factor, which predicts both effective and efficient technology usage, is the acceptance of the innovative assistive working system. A working system is perceived to be useful, cover human needs, capabilities and expectations only if acceptance scores are high. Therefore, human-robot collaboration acceptance should be evaluated with regard to differences in the predictors of acceptance depending on the cross-cultural background of subjects from Germany, Japan, China, and the USA.

\subsection{The Present Study}

The aim of our research was to build an acceptance model with regard to human-robot collaboration that builds on already existing knowledge and takes context-specific factors of the interaction between human and robots in an industrial setting into account. Therefore, the model was developed over four consecutive stages.

First, a research model based on the literature was developed and reviewed in a workshop with associates of robot manufacturing companies, associates of companies that use industrial robots, employees working with robots, and scientists in the fields of psychology, computer science and engineering. This model took variables of the traditional technology acceptance models, such as TAM [10], TAM 2 [12] and TAM 3 [13] into account and was extended with regard to factors which came up during the workshop. As such, the model contains context-specific factors that might be the subject for adaptation in work systems, such as perceived enjoyment, perceived safety, ethical, legal and social implications and, on the other hand, personal characteristics such as self-efficacy, robot anxiety, affinity towards technology (adapted from [33]) and perceptions of external control, which are considered as variables with uncertain influence on the predictors. The ELSI factors were added due to the context of human-robot collaboration (e.g. "I fear that I lose the contact to my colleagues because of the robot"). ELSI deal, among other things, with the advantages and disadvantages of the technology. On the one hand, employees can be supported in physically demanding or monotonous tasks. On the other hand, the growth of robot systems can lead to job losses and a decline in human skills and knowledge. This dualism of technology leads to the need to consider ethical, legal and social implications in the development of human-robot systems. [34]. As a second step, a survey based on the emerged variables was developed and iteratively validated with experts. Third, the survey was implemented in form of an online tool and completed by 1326 participants. The participants were recruited according to the chosen countries with the support of a specialized survey panel company. Participants with at least 1 month of professional working experience as operational production workers were selected for the study. Lastly, the model was analyzed statistically by correlation analyses in order to draw conclusions with regard to possible predictors concerning the acceptance of robots.

The different taxonomies of human-robot collaboration and robots are complex and can be divided into communication channel, robot task, physical and temporal proximity, kind of collaboration, field of application, robot morphology, human interaction role, degree of robot autonomy and team composition [35]. In this study, the role of the robot in the human-robot system is presented as active and passive in a simplified way. As robots can adopt an active role (e.g. handing over heavy components) or a passive role (e.g. hold a component so that the human can work on that component), we built two scenarios for the survey in order to make predictions concerning both ways of interacting. Participants were instructed to base their response behavior on the scenario including the robot as an active partner for interaction or as a passive partner for interaction.

\section{Method}

\subsection{Participants}

Altogether, $N=1326$ subjects completed the online survey of the study, recruited via a panel survey $(N=322$ from Germany, $N=360$ from Japan, $N=349$ from China and 


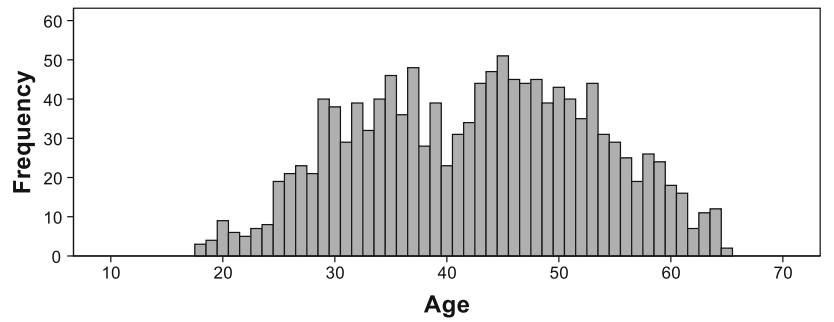

Fig. 1 Histograms of the study's age distribution

$N=295$ came from the USA). All of them were working in manufacturing. Gender was balanced but representative for production employees: $74.9 \%$ of the survey participants were male and $25.1 \%$ were female. Ages ranged from 18 to 65 years $(M=42.6, S D=10.5)$. Figure 1 shows a histogram of the study's age distribution. For the evaluation only completed questionnaires were considered.

In the context of conducting this study, the Ethics Committee at the RWTH Aachen School of Medicine discussed the ethics proposal and assessed it as follows: "From an ethical and professional law perspective, there are no concerns about the research project" (EK 013/16).

\subsection{Instrument}

The specific items of the survey are presented in Table 1 and include the reference to the original research except for questions related to ELSI that the researchers of this study developed, as described according to the procedure in the previous section. All items developed in the corresponding language were deemed to be adequate for the survey participants. As the researchers who conducted the study were native German speakers, the questionnaire was first developed in German. As a next step, a forward translation was carried out (i.e. the questionnaire was translated by a bilingual translator able to both speak German and the target language of the questionnaire). Third, a back translation was administered. Using the same approach as in the forward

Table 1 Items of the study

\begin{tabular}{|c|c|c|}
\hline & Factors & Items \\
\hline \multirow[t]{4}{*}{ TAM (Davis [10]) } & Perceived usefulness & Using the robot improves my performance in my job \\
\hline & Perceived ease of use & My interaction with the robot is easy \\
\hline & Behavioral intention & $\begin{array}{l}\text { If I could choose whether the robot supports me at work, I } \\
\text { would appreciate working with the robot }\end{array}$ \\
\hline & Use behavior & I prefer the robot to other machines in the industrial environment \\
\hline \multirow[t]{5}{*}{ TAM 2 (Venkatesh and Davis [12]) } & Subjective norm & In general, the organization supports the use of the robot \\
\hline & Image & $\begin{array}{l}\text { People in my organization who use the robot have more prestige } \\
\text { than those who do not }\end{array}$ \\
\hline & Job relevance & The use of the robot is pertinent to my various job-related tasks \\
\hline & Output quality & The quality of the output I get from the robot is high \\
\hline & Result demonstrability & $\begin{array}{l}\text { I have no difficulty telling others about the results of using the } \\
\text { robot }\end{array}$ \\
\hline \multirow[t]{4}{*}{ TAM3 (Venkatesh and Bala [13]) } & Perceived enjoyment & I find using the robot to be enjoyable \\
\hline & Self-efficacy & I can use the robot if someone shows me how to do it first \\
\hline & Robot anxiety & Robots make me feel uncomfortable \\
\hline & Perception of external control & I have the resources necessary to use the robot \\
\hline \multirow[t]{5}{*}{ ELSI } & Social implication & $\begin{array}{l}\text { I fear that I will lose the contact to my colleagues because of the } \\
\text { robot }\end{array}$ \\
\hline & Legal implication (occupational safety) & I do not mind if the robot works with me at a shared workstation \\
\hline & Legal implication (data protection) & I do not mind if the robot records personal information about me \\
\hline & Ethical implication & I fear that I will lose my job because of the robot. \\
\hline & Perceived safety & I feel safe while using the robot \\
\hline Other & Technology affinity (Karrer et al. [33]) & $\begin{array}{l}\text { I like to visit shops for electronic devices } \\
\text { Electronic devices lead to intellectual impoverishment } \\
\text { Electronic devices make things cumbersome } \\
\text { I inform myself about electronic devices, even if I do not have } \\
\text { the intention to purchase them } \\
\text { Electronic devices make people independent } \\
\text { Trying new electronic devices is fun } \\
\text { I know most of the functions of the devices I own }\end{array}$ \\
\hline
\end{tabular}


Table 2 Structure of the online survey

\begin{tabular}{|c|c|}
\hline Part & Factors/information \\
\hline \multirow[t]{2}{*}{ Introduction } & Project aim and funding \\
\hline & Call for participation \\
\hline Anchor variables & $\begin{array}{l}\text { Self-efficacy, robot anxiety, } \\
\text { technology affinity, } \\
\text { perceptions of external } \\
\text { control }\end{array}$ \\
\hline Scenario description & $\begin{array}{l}\text { Active robot, passive robot } \\
\text { (permuted) }\end{array}$ \\
\hline $\begin{array}{l}\text { Adaptation variables (for each } \\
\text { scenario) }\end{array}$ & $\begin{array}{l}\text { Perceived enjoyment, social } \\
\text { implication, legal } \\
\text { implications, ethical } \\
\text { implications, perceived safety }\end{array}$ \\
\hline $\begin{array}{l}\text { Other variables (for each } \\
\text { scenario) }\end{array}$ & $\begin{array}{l}\text { Subjective norm, image, job } \\
\text { relevance, output quality, } \\
\text { result demonstrability, } \\
\text { perceived usefulness, } \\
\text { perceived ease of use, } \\
\text { behavioral intention, use } \\
\text { behavior }\end{array}$ \\
\hline Demographical data & Gender and age \\
\hline Conclusion & $\begin{array}{l}\text { Possibility for final comments } \\
\text { on human-robot collaboration } \\
\text { and comments on the survey }\end{array}$ \\
\hline
\end{tabular}

translation, the questionnaire was given to an independent translator who spoke the questionnaire language and German in order to find any discrepancies in the translation. In case the translation was regarded as equivalent and properly translated, the questionnaire was used for the study. Otherwise, the procedure was repeated for critical terms in the questionnaires. The participants rated the degree of consent with the statements on a 7-point Likert scale, with $1=$ fully disagree and $7=$ fully agree.

\subsection{Procedure}

The survey was structured into explanatory parts, such as the project description and questionnaire parts, e.g. for the variables of the model (see Table 2). The software used for the online survey was Unipark, the academic program of Questback. The two scenarios were described as follows:

Active robot: "You are an order picker, working with a robot arm at a shared workstation. Your job is to take a case and the corresponding snap lid and hold these components compatible between you and the robot arm onto the working plate. The robot takes the corresponding screws one after the other and fastens the snap lids to the housing. After mounting the components, the robot puts the constructed part in the order picking area next to the workstation and a new process begins."
Passive robot: "You are an order picker, working with a stationary robot arm at a shared workstation. The robot takes an automobile door from the reserve storage and holds it in front of you. You then take individual car components from a shelf and mount these components on the automobile door. After the car components are mounted, the robot puts the door in the order picking area next to the workstation and a new process begins."

\subsection{Statistical Analysis}

Correlation coefficients were used to determine the relationships in our model and were calculated by using Spearman's rho. According to Cohen [36], effect sizes can be classified into low $(r=.10)$, medium $(r=.30)$ and large $(r=.50)$. The level of significance was set to $\alpha=.05$. The complete HRC acceptance model based on the accumulated data from Germany, Japan, China and the USA is presented in Fig. 2.

\section{Results}

The HRCAM for all four countries is described in more detail in the following sections. Regarding perceived usefulness, the most important predictor in the robot acceptance model is job relevance, followed by subjective norm, which might be caused by the job-related and industrial context of the model.

Perceived ease of use is influenced by the anchor variables and the adjustment variables. Regarding the anchor variables, the highest correlation coefficients were found for the variables of the traditional TAM 3 model self-efficacy, robot anxiety and perceptions of external control. Technological affinity was negatively correlated with perceived ease of use.

Concerning the variables for adjustment, it was shown that perceived enjoyment, perceived safety and occupational safety are the best predictors for perceived ease of use showing high correlation coefficients, whereas social and ethical implications are less important as they show correlation coefficients which can be classified as medium.

Altogether, correlation coefficients between perceived usefulness, perceived ease of use, behavioral intention and use behavior reached medium to high levels, showing that the original model is transferrable to the domain of human-robot interaction.

The robot acceptance models for the individual countries of Germany, Japan, China and the USA can be found in the Appendix under Figs. 3, 4, 5, and 6. The differences between the overall robot acceptance model and the individual models worth highlighting are briefly explained below. 


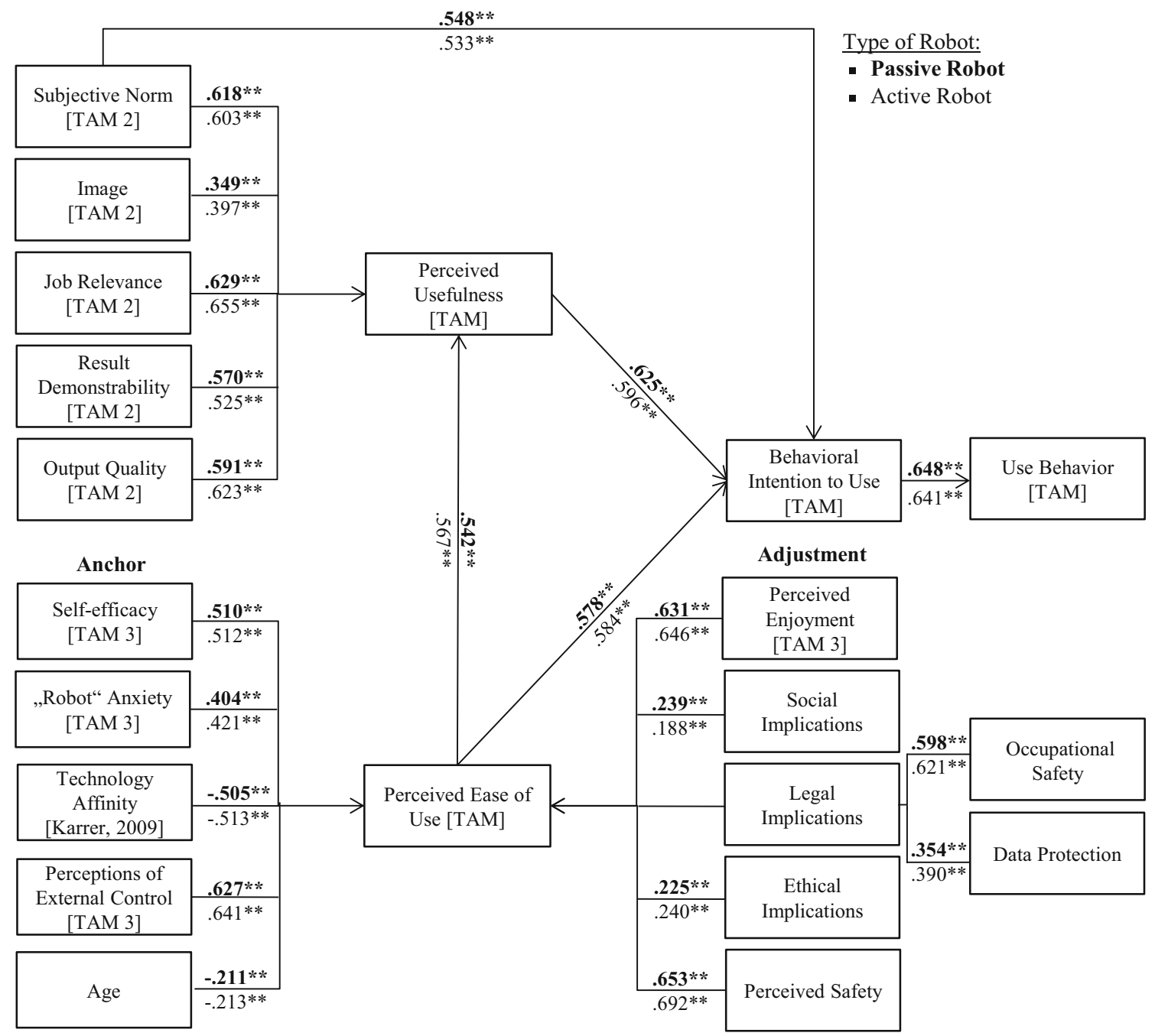

Fig. 2 HRCAM with correlation coefficients as strength of associations, ${ }^{*} p<.05$, ** $p<.001,(N=1326)$

\subsection{TAM in Germany}

The acceptance model of the German sample is shown in Fig. 3 of the Appendix. It is noticeable that the correlation coefficients for both active and passive robot types are large $(r>.50)$ for the variables of subjective norm, job relevance, perceptions of external control, perceived safety and occupational safety. The highest correlation coefficient in the model is found between job relevance and perceived usefulness for the active type of robot, implying that job relevance is the greatest predictor for perceived usefulness. For the variables of result demonstrability, self-efficacy, robot anxiety, social implications and data protection, the correlation coefficients vary between medium and large $(.30<r<.50)$ for both active and passive types of robots, whereby the correlation coefficient for self-efficacy and output quality are highest. The lowest correlation coefficients in the model are found between image and perceived usefulness and between ethical implications and perceived ease of use.

In general, it is noticeable that correlation coefficients are on average smaller for the German sample compared to the overall model. The greatest difference between the models is detectable between the variables of age, technological affinity and image.

\subsection{TAM in Japan}

The results of the sample from Japan are shown in Fig. 4 of the Appendix. The most striking finding about technology acceptance in Japan is the fact that the correlation coefficients for ethical implications and the perceived ease of use are much smaller $(r<.10)$ compared to the other analyzed countries. Furthermore, it is noticeable that the correlation coefficients for both active and passive robot types are large 
for the variables of subjective norm, job relevance and result demonstrability, whereby the correlation coefficient between result demonstrability and perceived usefulness is the greatest predictor in the model-as was already found in the German sample. For the variables of perception of external control and perceived safety, the correlation coefficients are large for the active or passive robot type; for the passive robot type, the correlation coefficients are between medium and large. For the variables of output quality, self-efficacy, perceived enjoyment, occupational safety and data protection, the correlation coefficients are between medium and large for the active and passive robot type.

Generally, comparing Japan and the overall model shows that the correlation coefficients for the variable of job relevance are noticeably higher for Japan and significantly lower for the variables of self-efficacy, robot anxiety, technology affinity, perceived enjoyment, social implications and ethical implications.

\subsection{TAM in China}

The results of the sample from China are shown in Fig. 5 of the Appendix. The greatest correlation coefficient is found between perceived enjoyment and perceived ease of use, followed by the correlation coefficient between output quality and perceived usefulness. In addition, it is noticeable that the effect sizes for both active and passive robot types are large for the variables of subjective norm, result demonstrability, perception of external control, perceived enjoyment, perceived safety, occupational safety and data protection. For the variables of image, job relevance, self-efficacy, robot anxiety and ethical implications, the effect sizes are between medium and large for the active and passive robot type.

Comparing China and the overall model, the effect sizes for the variables of image and data protection are noticeably higher for China and significantly lower for the variables of job relevance and self-efficacy.

\subsection{TAM in the USA}

The results of the subjects from the USA are shown in Fig. 6 of the Appendix. It is noteworthy that the correlation coefficients are highest for perceived enjoyment and perceived ease of use as well as output quality and perceived usefulness. Furthermore, it should be noted that the correlation coefficients for both active and passive robot types are large for the variable subjective norm, job relevance, perception of external control, perceived safety and occupational safety. For the variable of result demonstrability, the correlation coefficients are large for the passive robot type; for the active robot type, the effect sizes are a little lower than large and between medium and large. For the variables of image, self-efficacy and data protection, the effect sizes are between medium and large for the active and passive robot type.

Comparing the model of the USA and the overall model, the correlation coefficient between robot anxiety and perceived ease of use are noticeably higher and significantly lower between self-efficacy and perceived ease of use.

\subsection{Cross-Cultural Comparison of TAM}

The cross-cultural differences between Germany, the USA, Japan and China are shown in Table 3. In the following, striking cultural differences in robot acceptance are mentioned. The greatest cross-cultural differences between the four countries considered are in the variables job relevance, technology affinity, perceived enjoyment, social implications, data protection and ethical implications.

For the variable job relevance, the correlation coefficients for China deviate noticeably from the other three cultures, especially compared to Germany and Japan. Regarding technology affinity, the correlation coefficients for China differ from the other three cultures, with the greatest differences compared to Germany and Japan. Further cultural differences are notable in the variables of perceived enjoyment, social implications, data protections, and ethical implications. For the variable of perceived enjoyment, the correlation coefficients for Japan differ from Germany and the USA in particular. With regard to the variable of social implications, the correlation coefficients for Germany differ above all from those for the USA and Japan. For data protection, the correlation coefficients for Germany differ mainly from those for China. For ethical implications, the correlation coefficients for Japan differ considerably, especially from those for China.

\section{Discussion}

\subsection{Comparison to Historical Technology Acceptance Models}

A comparison between the results from the basic technology acceptance model (TAM 1) according to $10[10,11]$ and the human-robot collaboration acceptance model presented in this paper (with correlation coefficients as strength of associations between person-specific anchor variables, context-specific adjustment variables, and the target variable use behavior) from Fig. 2 shows that the basic direction of the significant effects is consistent. In addition, it is striking that the effect size between perceived ease of use and perceived usefulness is large $(r>.50)$ for both models. Moreover, the 
Table 3 Cross-cultural

differences in robot acceptance for Germany, Japan, the

People's Republic of China and the United States of America

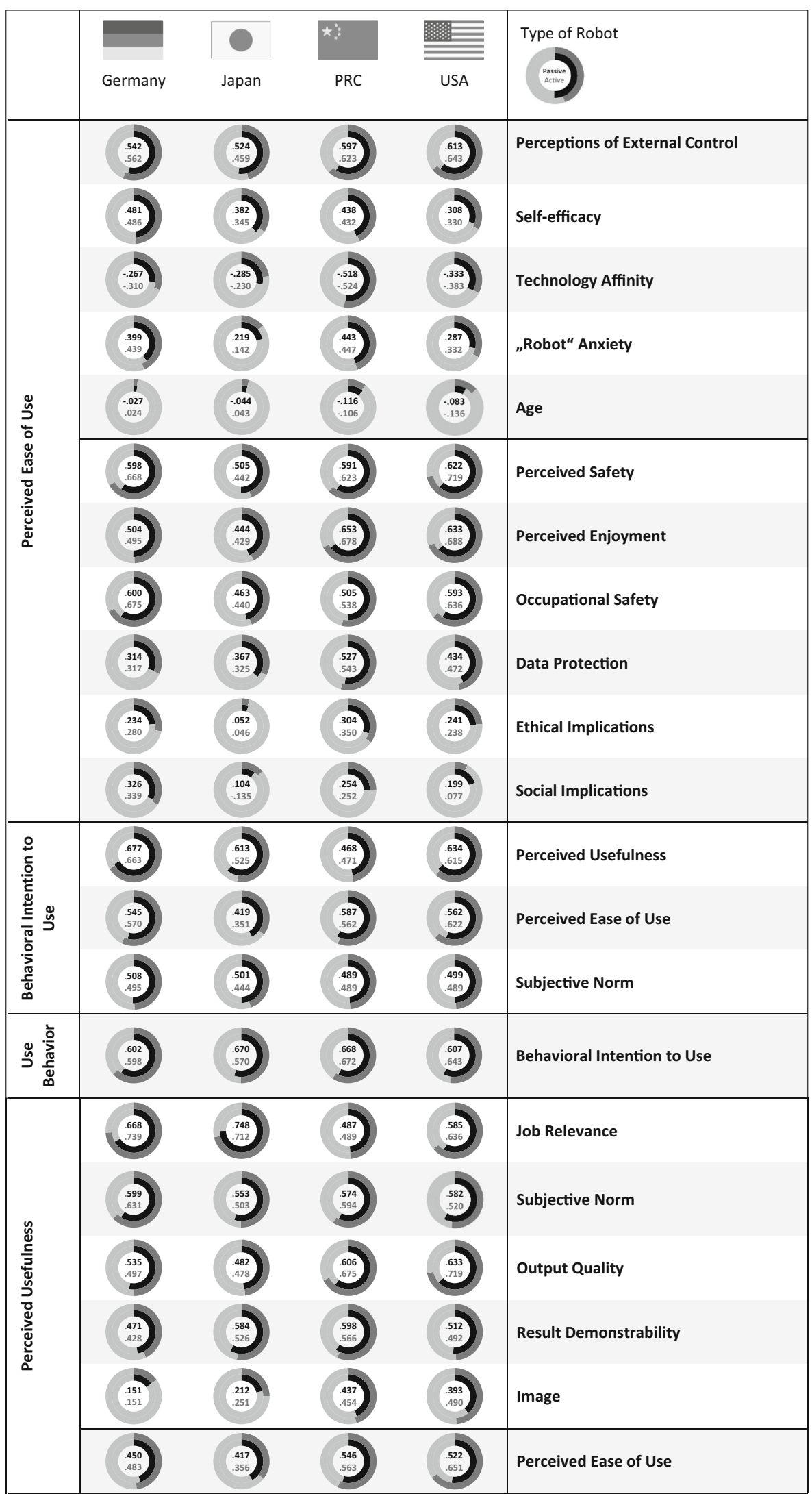


effect size of the correlation between perceived usefulness and behavioral intention to use for the HRCAM is large. Analogously, the correlation in the TAM 1 between perceived usefulness and intention to use is large. It also appears for the HRCA model that perceived ease of use has a large and significant effect on behavioral intention to use. This is analogous to the context in the TA model that perceived ease of use has a smaller but nonetheless significant effect on attitude towards using. This shows that TAM 1 can be used as a starting point for developing the human-robot collaboration acceptance model [10, 11].

Furthermore, a comparison between the second technology acceptance model (TAM 2) according to 12 [12], the third technology acceptance model (TAM 3) according to 13 [13] and the human-robot collaboration acceptance model presented in this contribution shows that the direction of the correlations are the same. These similarities with the already sufficiently validated historical models (TAM 1-3) shows that the fundamental assumption of technology acceptance can be applied to the context of robotics.

\subsection{Differences Between Active and Passive Robot Types}

For the two scenarios - the robot in the role of active and passive collaboration partner-the correlation coefficients representing the strength of association between person-specific anchor variables, context-specific adaptation variables, and the target variable use behavior differed in several respects.

For the variables of image (passive robot: .349/active robot: .397), output quality (.591/.623), perceived safety (.653/.692), and data protection (.354/.390), the correlation coefficients between the robot in the passive and the active role differ noticeably (by more than $\Delta=.03$ ), with lower values for the passive robot.

For the variables of result demonstrability $(.570 / .525)$ and social implications (.239/.188), the correlation coefficients between the robot in the passive and the active role differ markedly, with lower values for the active robot.

Initially, as already mentioned in Chapter 1.2, differences regarding an active and passive robotic type could be expected (Nomura et al. [30, 31], Lee et al. [32]). However, neither the results of this study regarding the robot in an active role compared to the robot in a passive role nor the above-mentioned literature show large differences, suggesting that either the area of operation of robots can be ignored when modeling acceptance or that our scenarios did not differ sufficiently.

\subsection{Human-Robot Collaboration Acceptance Model}

Regarding perceived usefulness, the most important predictor in our model is job relevance (.629/.655), followed by subjective norm, output quality, and result demonstrability. A possible explanation for this is the industrial context of the model.

Regarding the anchor variables, we found the highest correlation coefficients for the variables of the traditional TAM 3 perceptions of external control, self-efficacy, and robot anxiety. Technology affinity was negatively correlated with perceived ease of use. Possible reasons for the negative correlation of technological affinity and ease of use may be that persons with a higher technological affinity have more information and technical expertise and therefore are better informed about the complexity of technology. Thus, they are more likely to know the vulnerabilities of novel systems. From this, it can be assumed that persons with a higher technological affinity have a more distinct opinion and higher expectations about human-robot collaboration scenarios. With regard to anchor variables and physiological factors such as age, it should be noted that these are personrelated or individual and cannot simply be influenced.

In contrast, the adjustment variables can be positively influenced by the ergonomic criteria and the aspect of robot acceptance. The adjustment variables of perceived enjoyment, perceived safety and occupational safety are the best predictors for the perceived ease of use showing high effectsizes, whereas social implications, data protection and ethical implications are of lesser relevance showing medium effect sizes. It follows that the adjustment variables, in particular perceived enjoyment, perceived safety and occupational safety, should be given special attention in the design of human-robot collaboration. Through a design that takes ergonomics and user experience into account, perceived ease of use and thus behavioral intention to use can be positively influenced.

For example, an ergonomic design according to ISO 9241-210 can increase the usability or user experience of human-robot collaboration (effectiveness, efficiency, satisfaction) and thus perceived enjoyment. By means of an ergonomic design of the adjustment variables, perceived ease of use and hence behavioral intention to use and the use behavior of human-robot collaboration can be increased.

Overall, correlation coefficients between perceived usefulness, perceived ease of use, behavioral intention and use behavior reached high levels, indicating that the original technology acceptance model is transferrable to the domain of human-robot collaboration.

\subsection{Cross-Cultural Differences in Robot Acceptance}

In addition, striking cultural differences in robot acceptance (more than $\Delta=.2$ between the highest and lowest correlation coefficients for the robot in the role of active and passive interaction partner) are discussed on the basis of Table 3. 
Regarding cultural differences for perceived usefulness, the most substantial finding is job relevance. For the variable of job relevance, the correlation coefficients for China (.487/.489) deviate noticeably from the other three cultures, the biggest difference being compared to Germany (.748/.712) and Japan (.668/.739). One possible explanation for this is that the level of automation in China is lower than in Germany and Japan.

In terms of the anchor variables, which refer to perceived ease of use, it is striking that for technology affinity the correlation coefficients for China $(-.518 /-.524)$ differ from those of the other three cultures, with the greatest differences compared to Germany $(-.267 /-.310)$ and Japan ($.285 /-.230$ ). One possible reason for this difference is that in China, the spread and use of technology in everyday life, such as smartphones, has increased considerably in recent years across all ages, and smartphones with various apps are used for cashless payments in everyday situations such as shopping.

With a view to the adjustment variables, which also relate to perceived ease of use, cultural differences are notable in the variables of perceived enjoyment, social implications, data protection, and ethical implications. For the variable of perceived enjoyment, the correlation coefficients for Japan (.444/.429) differ from those for China (.653/.678) and the USA (.633/.688) in particular. This lower correlation coefficient between perceived enjoyment and perceived ease of use for Japan could be caused by a high habituation effect and the immense spread of robots in everyday life as in restaurants and hotels in Japan. With regard to the variable of social implications, the correlation coefficients for Germany (.326/.339) differ above all from those for the USA (.199/.077) and Japan (.104/- .135). The fear of losing contact with colleagues in the future due to the robot may be smaller in Japan than in other countries, because robots are already widespread in Japan. The correlation coefficients between data protection and perceived ease of use for Germany (.314/.317) differ mainly from those for China (.527/.543). The correlation coefficients between data protection and perceived ease of use may be comparatively low for Germany due to the extensive data protection laws and comparatively high for China due to the extensive spread of video surveillance. The correlation coefficients between ethical implications and perceived ease of use differ considerably between Japan (.052/.046) and China (.304/.350). Two explanations for this are the already high level of automation and the positive connotation of automation in Japan, which may lead to a low fear of losing the job due to a robot.

The studies presented in the literature review in Chapter 1.2 focus only on individual factors such as, for example, style of communication, attitudes towards robots or trust, neither of which are according to TAM 3 or the technology acceptance model regarding human-robot collaboration presented in this paper (see Fig. 2). Therefore, the findings in the literature regarding cultural differences and the cultural differences in robot acceptance illustrated in Table 3 are not directly comparable.

On the basis of the results, the examined countries cannot be classified as "western" or "eastern" cultures. In addition, the potential causes for differences in technology acceptance between the countries under consideration are not mono-causal and cannot be explained in general terms by cross-cultural differences between West and East. Rather, possible causes such as level of automation and positive connotations of automation, spread and use of technology in everyday life, habituation effects and spread of robots, data protection laws versus spread of video surveillance indicate that there are multiple causes of difference, including social and political differences.

\subsection{Limitations}

The findings of this study should be interpreted in light of certain limitations, however. First, the developed models, although they involved participants of four different cultures, are not globally valid, as they are restricted to the analyzed cultures. Further research would be needed in order to make more precise predictions regarding other cultures.

Another limitation regarding the classification of the sample of the study concerns the fact that a panel company was commissioned to select the subjects. The aim of a sample is always to be able to make statements about the population based on an analysis of a sample. In a representative sample, the results of the panel participants can be extrapolated to the respective population without systematic errors. If a non-representative sample is used, the results are distorted and extrapolation is not possible. Distortion may be caused by the non-participation of selected persons or by incorrect sample selection.

The problem of lack of representativeness as a result of selection processes can have two causes. Self-selection occurs when the initiative to participate comes from the participants themselves and not from the organization conducting an online panel. Such a sample is distorted in the sense that the people who consciously decide to participate usually overrepresented certain groups of people. The population of online panels can also lead to distortions, as it consists only of Internet users. Without Internet access, no one can take part in the studies, so that a large part of the total population is excluded from the outset. These are factors which might have an influence on the panel that was selected in order to build the technology acceptance models 
and which needs to be considered when using panel companies in order to select samples.

A last limitation that is worth mentioning deals with the translation of the questionnaires that were used to build the technology acceptance models. As was described in more detail in the method section of the paper, a forward and back translation were administered in order to translate the German questionnaire to the three other languages. However, this procedure does not rule out the possibility of having improperly translated words or terms in the questionnaire that, in the worst case, miss the intended meaning.

\section{Conclusion}

Basically, this contribution shows that the historical findings on technology acceptance research, which are based on human-computer interaction, can also be applied to human-robot collaboration with the appropriate adaptations. With knowledge of the variables that influence behavioral intention to use robots, the interaction with robots can be positively influenced.

Anchor variables are person-specific and cannot be changed in the short or medium term, but a corresponding corporate management can promote robot acceptance in the long term, for instance. The factor perceptions of external control ("The degree to which an individual believes that organizational and technical resources exist to support the use of the system") from TAM 3 [13] is an example of an anchor variable that can be influenced by corporate management to change the attitude of employees in the long term.
On the other hand, adjustment variables can be positively influenced in the short to medium term. The factor of perceived safety ("I feel safe while using the robot") is an example of such an adjustment variable. This can be done either preventively when the development team designs the innovative assistive working system or reactively when corporate management takes the suitable technical, organizational and personal measures. Beyond that, scientific and practical findings on industrial engineering, ergonomic design and occupational health and safety can be helpful. Among other things, the design requirements and protective measures of relevant standards such as 4 must be taken into account. This can be supplemented by information on crosscultural differences of acceptance.

Acknowledgements This publication is part of the "MeRoSy" research project funded by the German Federal Ministry of Education and Research (BMBF, Grant No. 16SV7190).

Funding This study was funded by the German Federal Ministry of Education and Research (BMBF, Grant No. 16SV7190).

\section{Compliance with Ethical Standards}

Conflict of interest The authors declare that they have no conflict of interest.

Open Access This article is distributed under the terms of the Creative Commons Attribution 4.0 International License (http://creativecomm ons.org/licenses/by/4.0/), which permits unrestricted use, distribution, and reproduction in any medium, provided you give appropriate credit to the original author(s) and the source, provide a link to the Creative Commons license, and indicate if changes were made.

\section{Appendix}

See Figs. Figures 3, 4, 5, and 6. 


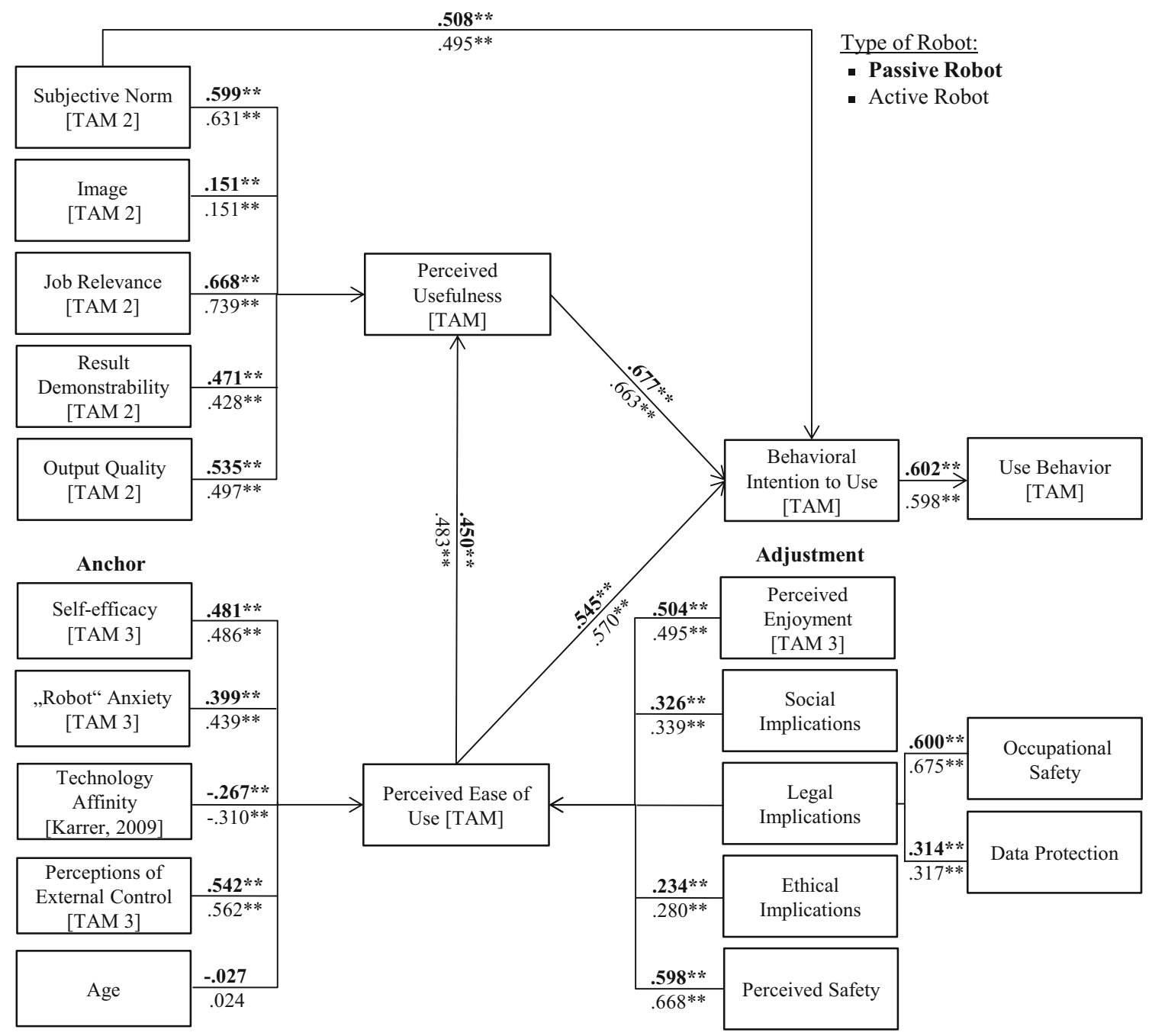

Fig. 3 Robot acceptance model for Germany with correlation coefficients as strength of associations between general acceptance variables, personspecific anchor variables, age and context-specific adjustment variables, on the target variables, ${ }^{*} p<.05, * * p<.001,(\mathrm{~N}=322)$ 


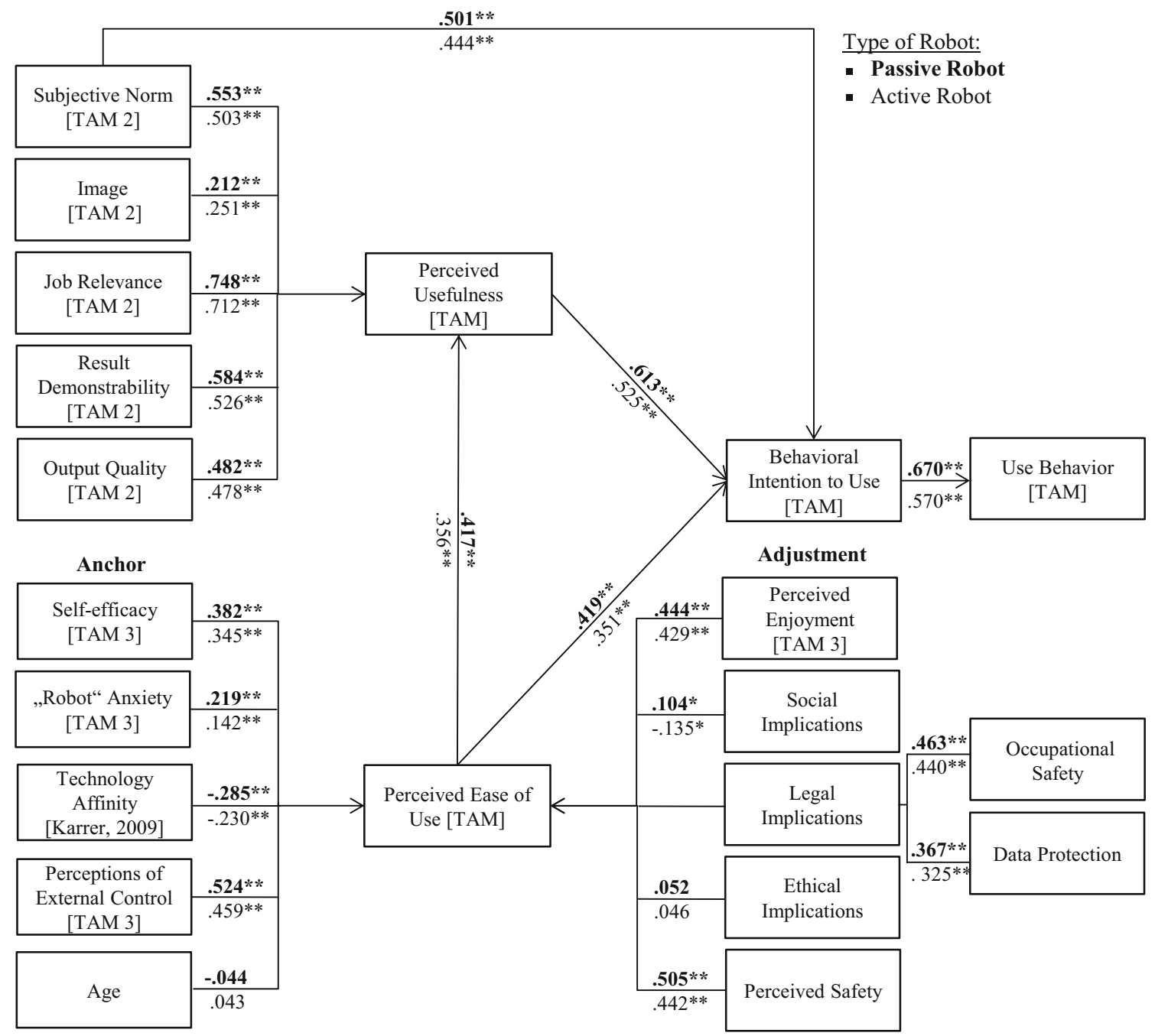

Fig. 4 Robot acceptance model for Japan with correlation coefficients as strength of associations between person-specific anchor variables, contextspecific adjustment variables, age and the target variable use behavior, $* p<.05, * * p<.001,(\mathrm{~N}=360)$ 


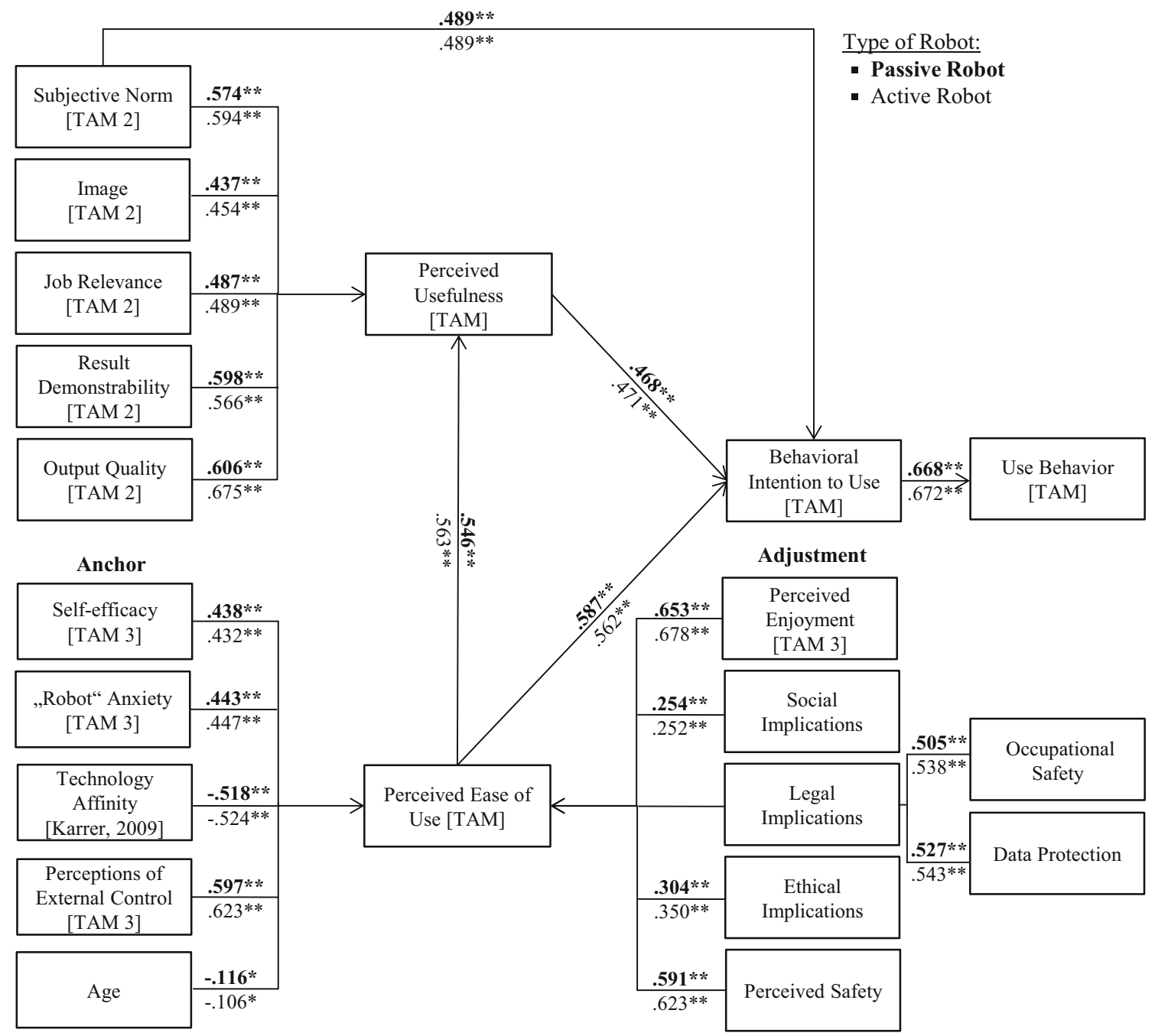

Fig. 5 Robot acceptance model for the People's Republic of China with correlation coefficients as strength of associations between person-specific anchor variables, context-specific adjustment variables, age and the target variable use behavior, $* p<.05, * * p<.001,(\mathrm{~N}=349)$ 


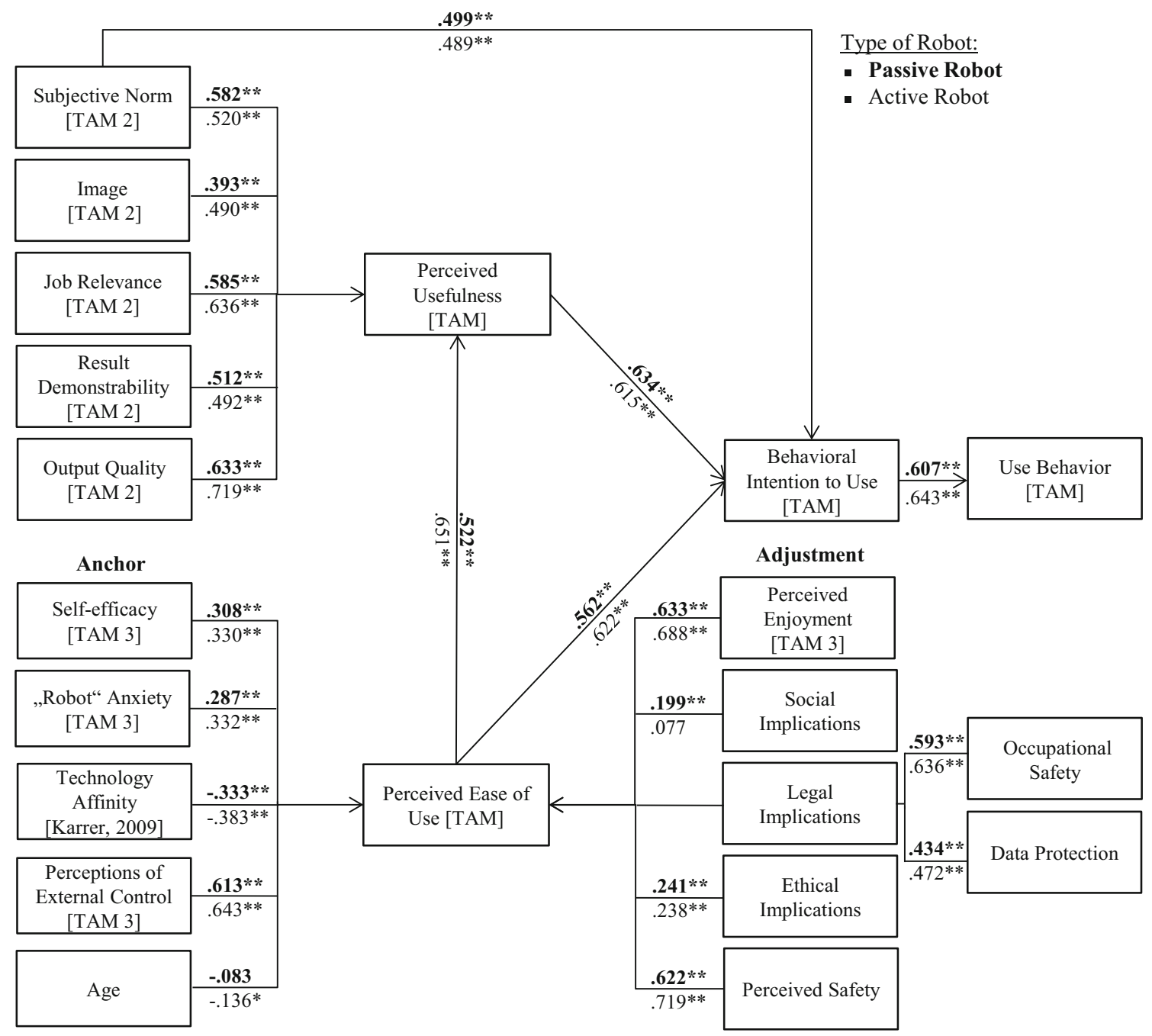

Fig. 6 Robot acceptance model for the United States of America with correlation coefficients as strength of associations between person-specific anchor variables, context-specific adjustment variables, age and the target variable use behavior, $* p<.05, * * p<.001,(\mathrm{~N}=295)$

\section{References}

1. Ray C, Mondada F, Siegwart R (2008) What do people expect from robots? In: 2008 IEEE/RSJ international conference on intelligent robots and systems. IEEE, pp 3816-3821

2. Kopacek P (2013) Development trends in robotics. Elektrotech Inftech 130(2):42-47. https://doi.org/10.1007/s00502-013-0129-1

3. Brandl C, Mertens A, Schlick CM (2016) Human-robot interaction in assisted personal services: factors influencing distances that humans will accept between themselves and an approaching service robot. Hum Factors Manuf 26(6):713-727. https://doi.org/10. 1002/hfm.20675

4. International Standardization Organization (2012) Robots and robotic devices - safety requirements for industrial robots-Part 1: robots (ISO 10218-1)

5. International Standardization Organization (2016) Robots and robotic devices-collavorative robots(DIN ISO/TS 15066, DIN SPEC 5306)

6. IFR (2016) Internatinaler vergleich der roboter-dichte. https://de.statista.com/infografik/4662/roboter-automatisierunginternational/. Accessed 19 Sept 2017
7. IFR (2017) Absatz von industrierobotern nach ausgewählten ländern weltweit im jahr 2015. https://de.statista.com/statistik/ daten/studie/188252/umfrage/einsatz-von-industrierobotern-ineuropa-nach-region/. Accessed 19 Sept 2017

8. IFR (2017) Geschätzter bestand von industrierobotern weltweit in den jahren 2010 bis 2019 (in 1.000 Stück). https://de.statista.com/ statistik/daten/studie/250212/umfrage/geschaetzter-bestand-vonindustrierobotern-weltweit/. Accessed 19 Sept 2017

9. Rogers EM (1962) Diffusion of innovations. Free Press of Glencoe, New York

10. Davis FD (1989) Perceived usefulness, perceived ease of use, and user acceptance of information technology. MIS Q 13(3):319. https://doi.org/10.2307/249008

11. Davis FD (1993) User acceptance of information technology: system characteristics, user perceptions and behavioral impacts. Int $\mathbf{J}$ Man Mach Stud 3:475-487

12. Venkatesh V, Davis FD (2000) A theoretical extension of the technology acceptance model: four longitudinal field studies. Manag Sci 46(2):186-204. https://doi.org/10.1287/mnsc.46.2.186.11926

13. Venkatesh V, Bala H (2008) Technology acceptance model 3 and a research agenda on interventions. Decis Sci 39(2):273-315. https:// doi.org/10.1111/j.1540-5915.2008.00192.x 
14. Hall ET (1989) Beyond culture. Anchor, Norwell

15. Evers V, Maldonado HC, Brodecki TL et al (2008) Relational vs. group self-construal. In: Fong T, Dautenhahn K, Scheutz M et al (eds) Proceedings of the 3rd international conference on Human robot interaction-HRI '08. ACM Press, New York, p 255

16. Lee HR, Sabanović S (2014) Culturally variable preferences for robot design and use in South Korea, Turkey, and the United States. In: Proceedings of the 2014 ACM/IEEE international conference on Human-robot interaction. pp 17-24

17. Trovato G, Zecca M, Sessa S et al (2013) Cross-cultural study on human-robot greeting interaction: acceptance and discomfort by Egyptians and Japanese. Paladyn J Behav Robot. https://doi.org/ 10.2478/pjbr-2013-0006

18. Wang L, Rau P-LP, Evers V et al (2010) When in Rome: the role of culture and context in adherence to robot recommendations. In: Proceedings of the 5th ACM/IEEE international conference on human-robot interaction. pp 359-366

19. Rau PP, Li Y, Li D (2009) Effects of communication style and culture on ability to accept recommendations from robots. Comput Hum Behav 25(2):587-595. https://doi.org/10.1016/j.chb.2008.12. 025

20. Mahadevan J (2013) Performing interplay through intercultural simulations. Int J Cross Cult Manag 13(3):243-263. https://doi. org/10.1177/1470595813507155

21. Bartneck C, Nomura T, Kanda T et al (2005) A cross-cultural study on attitudes towards robots. Hci international

22. Bartneck C, Suzuki T, Kanda T et al (2006) The influence of people's culture and prior experiences with Aibo on their attitude towards robots. AI Soc 21(1-2):217-230. https://doi.org/10.1007/ s00146-006-0052-7

23. Haring KS, Silvera-Tawil D, Matsumoto Y et al (2014) Perception of an android robot in Japan and Australia: a cross-cultural comparison. In: Beetz M, Johnston B, Williams M-A (eds) Social robotics, vol 8755. Springer, Cham, pp 166-175

24. Nomura TT, Syrdal DS, Dautenhahn K (2015) Differences on social acceptance of humanoid robots between Japan and the UK. In: Procs 4th int symposium on new frontiers in human-robot interaction

25. Li D, Rau PLP, Li Y (2010) A cross-cultural study: effect of robot appearance and task. Int J Soc Robot 2(2):175-186. https://doi.org/ 10.1007/s12369-010-0056-9

26. Haring KS, Mougenot $C$ et al (2014) Cultural differences in perception and attitude towards robots. IJAE 13(3):149-157. https:// doi.org/10.5057/ijae.13.149

27. Haring KS, Silvera-Tawil D, Takahashi T et al (2015) Perception of a humanoid robot: a cross-cultural comparison. In: 2015 24th IEEE international symposium on robot and human interactive communication (RO-MAN). IEEE, pp 821-826

28. van den Bergen B (2012) Differences between Germans and Dutch people in perception of social robots and the tasks robots perform. In: 16th Twente student conference on IT, Enschede, Netherlands

29. Conti D, Cattani A, Di Nuovo S et al (2015) A cross-cultural study of acceptance and use of robotics by future psychology practitioners. In: 2015 24th IEEE international symposium on robot and human interactive communication (RO-MAN). IEEE, pp 555-560

30. Nomura T, Kanda T, Suzuki $T$ et al (2007) Implications on humanoid robots in pedagogical applications from cross-cultural analysis between Japan, Korea, and the USA. In: RO-MAN 2007 - the 16th IEEE international symposium on robot and human interactive communication. IEEE, pp 1052-1057

31. Nomura T, Suzuki T, Kanda T et al (2007) What people assume about robots: cross-cultural analysis between Japan, Korea, and the USA. In: Human robot interaction. IntechOpen
32. Lee HR, Sung J, Sabanovic S et al (2012) Cultural design of domestic robots: a study of user expectations in Korea and the United States. In: 2012 IEEE RO-MAN: the 21st IEEE international symposium on robot and human interactive communication. IEEE, pp 803-808

33. Karrer K, Glaser C, Clemens C et al (2009) Technikaffinität erfassen-der Fragebogen TA-EG. Der Mensch im Mittelpunkt technischer Systeme 8:196-201

34. Nelles J, Kohns S, Spies J et al (2017) Best-practice approach for a solution-oriented technology assessment: ethical, legal, and social issues in the context of human-robot collaboration. In: Schlick CM, Duckwitz S, Flemisch F et al (eds) Advances in ergonomic design of systems, products and processes, vol 52. Springer, Berlin, Heidelberg, pp 1-14

35. Onnasch L, Maier X, Jürgensohn T (2016) Mensch-RoboterInteraktion-Eine Taxonomie für alle Anwendungsfälle. Bundesanstalt für Arbeitsschutz und Arbeitsmedizin, Dortmund

36. Cohen J (2013) Statistical power analysis for the behavioral sciences. Routledge

Publisher's Note Springer Nature remains neutral with regard to jurisdictional claims in published maps and institutional affiliations.

Dr. Christina Bröhl is a researcher at the Institute of Industrial Engineering and Ergonomics of RWTH Aachen University, Germany. Her research interests include cognitive engineering, technology acceptance, user experience design and human performance modeling. Dr. Bröhl's Ph.D. thesis aimed at understanding the cognitive mechanisms of human perception and attention. Specifically, she focused on identifying influences on perception in peripersonal space when interacting with technological devices.

Jochen Nelles received the B.E. degree in biomedical engineering in 2011 and the M.Sc. degree in industrial engineering in 2013 from the FH Aachen University of Applied Sciences, Germany. He is currently working towards the $\mathrm{Ph} . \mathrm{D}$. degree in mechanical engineering an RWTH Aachen University, Germany, in the field of human-robot interaction. His research interests are ergonomics/human factors and human-robot collaboration.

Dr.-Ing. Christopher Brandl studied Mechanical Engineering at the University of Stuttgart. At the RWTH Aachen University he received his doctorate in industrial engineering on the subject of ergonomic analysis of work-related postures. He gained practical experience in the industry at Daimler and Stihl. Since 2011, Christopher Brandl is working at the Institute of Industrial Engineering and Ergonomics of RWTH Aachen University. He is head of division ergonomics and human-machine-systems. His research interests are ergonomic analyses of manual work for work design.

Dr.-Ing. Dr. rer. medic. Dipl.-Inform. Alexander Mertens finished his master in Computer Science with focus on human-computer interaction and neurophysiology at RWTH Aachen University in 2008. In the year 2012 he finished his Ph.D. in Theoretical Medicine and in 2014 his Ph.D. in Engineering Sciences. Since 2015 he heads as Chief Engineer the "Ergonomics and Human-Machine Systems" department at the Institute of Industrial Engineering and Ergonomics (IAW). His research interests focus on designing target group specific user interfaces with an interdisciplinary inventory of methods. 
Verena Nitsch is currently professor and Director of the Institute of Industrial Engineering and Ergonomics at RWTH Aachen University. After studying Applied Psychology at Charles Sturt University in Australia and the University of Central Lancashire in the UK, she completed her Master's degree in Industrial and Organizational Psychology at Manchester Business School. From 2008 to 2013, she worked as a research assistant at the Human Factors Institute at the Bundeswehr University Munich, where she received her Doctorate of
Engineering in 2012 and was appointed assistant professor of Cognitive Ergonomics in October 2013. From 2016 to 2018 she was Acting Head of the Human Factors Institute of the Bundeswehr University Munich. Her research interests include human-machine interaction and user-centered technology development. 